\title{
Manufacturable Heterogeneous Integration for Flexible CMOS Electronics
}

\author{
Muhammad M. Hussain ${ }^{1}$, Sohail F. Shaikh ${ }^{1}$, Galo A. Torres Sevilla ${ }^{1,2}$, Joanna M. Nassar ${ }^{1,3}$, \\ Aftab M. Hussain ${ }^{1,4}$, Rabab R. Bahabry ${ }^{1,5}$, Sherjeel M. Khan ${ }^{1}$, Arwa T. Kutbee ${ }^{1,6}$, Jhonathan P. \\ Rojas $^{1,7}$, Mohamed T. Ghoneim ${ }^{1,8}$ and Melvin Cruz ${ }^{1}$ \\ ${ }^{1}$ King Abdullah University of Science and Technology, mmh Labs, Thuwal 23955-6900, Saudi Arabia \\ ${ }^{2}$ Swiss Federal Laboratories for Materials Science and Technology, Überland Str. 129, 8600 Dübendorf, \\ Switzerland \\ ${ }^{3}$ Department of Medical Engineering, California Institute of Technology, Pasadena, CA 91125, USA \\ ${ }^{4}$ International Institute of Information Technology, Hyderabad, Gachibowli, Hyderabad, Telangana 500032, India \\ ${ }^{5}$ King Fahd University of Petroleum and Minerals, Dhahran 31261, Saudi Arabia \\ ${ }^{6}$ King Abdulaziz University, Jeddah 21589, Saudi Arabia \\ ${ }^{7}$ MIT Media Lab, 77 Mass. Ave., E14/E15, Cambridge, MA 02139-4307 USA \\ Email: muhammad.hussain@kaust.edu.sa / Phone: +966 (544) 700-072
}

Nearly sixty years back when Jack Kilby built the first integrated circuit (IC), it was also the beginning of today's advanced and matured complementary metal oxide semiconductor (CMOS) technology whose arts and science of miniaturization has enabled Moore's Law to double up the number of devices in a given area in every two years. It has also been possible because CMOS technology has consistently adopted new materials and processes. High performance (data processing speed in computational devices), energy efficiency (for portable devices) and ultralarge-scale-integration (ULSI) density - all these features have been added to every major technology generation in additive manner. As we go forward and embrace Internet of Everything (IoE) where people, process, device and data are going to be seamlessly connected, we may want to ask ourselves a few fundamental questions about the future of CMOS electronics, enabling role of CMOS technology, potential benefits and application opportunities. Physically flexible electronics are increasingly getting attention as a critical and impactful expansion area for the general area of electronics. Many exciting demonstrations have been made to point out to its powerful prospect. Due to the paradox that traditional crystalline materials based electronics are useful in data management but they are naturally rigid and bulky, most of the researchers have resorted to two strategies: (i) non-silicon based fully flexible system with limited functionality and (ii) hybrid flexible electronic system with off-the-shelf ICs for data management. We do not consider this paradox is fundamental and a block-by-block approach using traditional CMOS technology can allow us to build fully flexible CMOS electronic systems [Fig. 1].

Our strategy includes [Fig. 2]: (i) use CMOS electronics when absolutely necessary (specially for data management: data processing, storage and communication); (ii) build the devices first on the crystalline substrates using state-ofthe-art CMOS technology to ensure high thermal budget compatibility and integration density; (iii) flexing those substrates with pre-fabricated devices - flexing has to be strategically designed to ensure higher area efficiency, stress management to maintain mechanical integrity, no performance and reliability compromise due to flexing processes [1, 2]; (iv) dicing the large flexible but fragile pieces using non-abrasive processes like laser ablation [3]; (v) transferring them on to destination site (soft substrates/encapsulation material) using automated, high-yield and reliable process; (vi) self-assembly based placement and bonding with higher yield without the need of traditional wire bonding (like Lego like lock-and-key assembly) [4]; (vii) drawing interconnects using inkjet printing and/or using low-cost flexible and stretchable but mechanically reliable emerging materials; (viii) completing the packaging using soft materials and potentially using an additive manufacturing method like 3D printing (while ensuring the active flexed die is placed in alignment with the neutral plane to enhance its mechanical lifetime) [5]; (ix) assembling interface electronics for data management on one side of the interposer soft substrates and integrating sensors and actuators on the other side of the same substrate like a coin to maximize utilization of sensing area, reducing heat dissipation induced effect on the sensing surface, faster data processing through shorter and denser interconnects which will be vertically aligned in different stages, same as in 3D ICs [6]; and (x) finally forming a through-polymer/silicon-via for out-of-plane interconnections. We have repeatedly demonstrated this is the most effective manufacturable heterogeneous integration strategy for fully flexible packaged multi-functional high-performance CMOS electronic systems.

References: [1] A. M. Hussain et al., Adv. Mater. DOI: 10.1002/adma.201504236, (2015). [2] R. R. Bahabry et al., Adv. Energy. Mater., DOI: 10.1002/aenm.201702221 (2018). [3] G. A. Torres Sevilla, A. Almuslem et al., Appl. Phys. Lett., vol. 108, p. 094102, (2016). [4] S. F. Shaikh et al., Adv. Mater. Tech. DOI: 10.1002/admt.201700147, (2017). [5] G. A. Torres Sevilla et al., Adv. Mater. Tech. DOI: 10.1002/admt.201600175, (2016). [6] A. M. Hussain et al., Small, vol. 12 (37), p. 5141, (2016). 


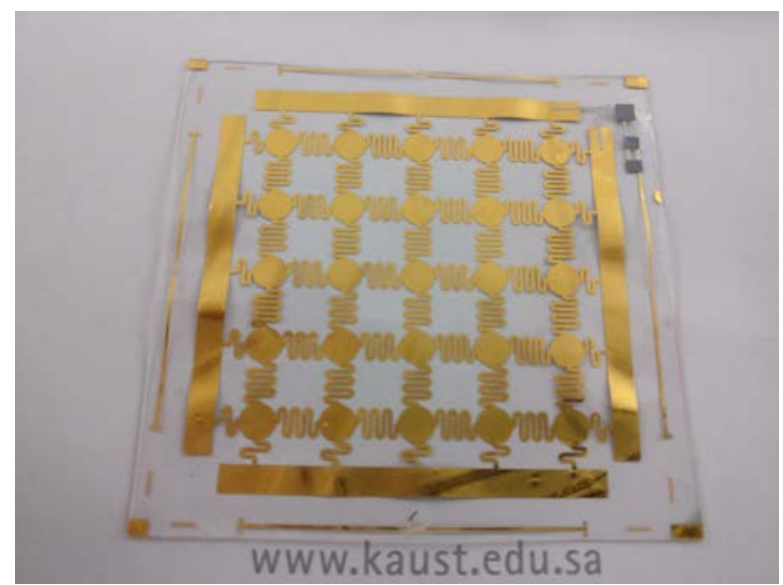

Fig. 1. A fully flexible CMOS electronic system with $16 \mathrm{MHz}$ microprocessor, 16 pin fan-out, built-in 512k flash memory and 4k DRAM integrated with 200 $\mathrm{mAh} / \mathrm{cm}^{3}$ capacity flexible micro-lithium-ion battery. The sensor array includes temperature, pressure, $\mathrm{pH}$ and humidty sensors.

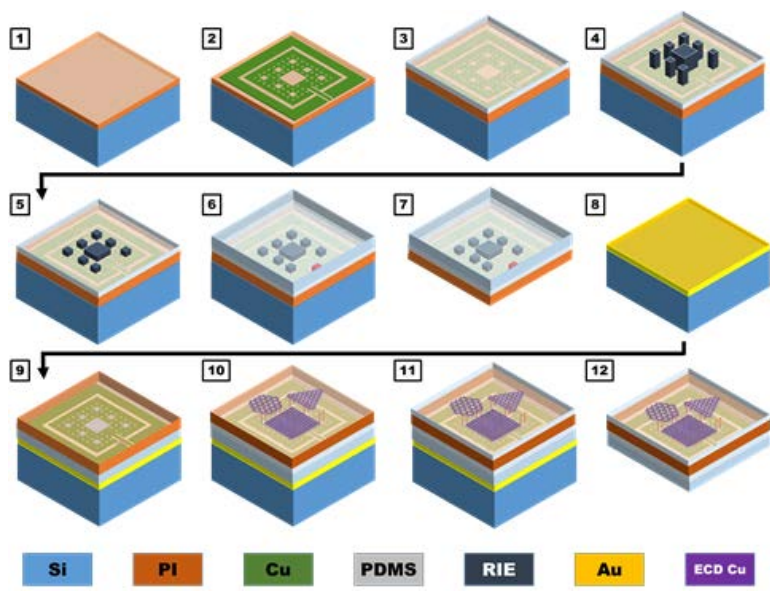

Fig. 2. Generic integration flow for fully flexible CMOS electronic system. The uniqueness of this flow is it allows interface data management electronics to be integrated on one side while the other side can host any sensor and actuators in material antagonistic way. 